
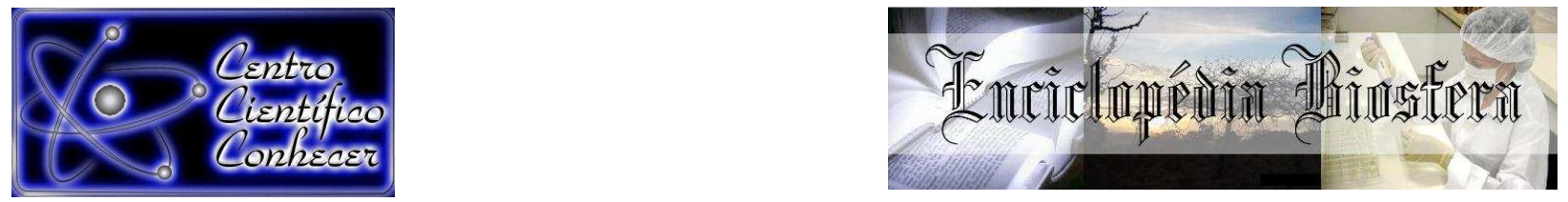

\title{
CONTRIBUIÇÃO TAXONÔMICA AO ESTUDO DO GÊNERO Bauhinia L. (LEGUMINOSAE) NO ESTADO DE MATO GROSSO, BRASIL
}

\author{
Juliete Vieira da Silva ${ }^{1}$, Célia Regina Araújo Soares Lopes ${ }^{2}$, José Martins \\ Fernandes $^{3}$ \\ ${ }^{1}$ Graduanda em Licenciatura Plena em Ciências Biológicas pela Universidade do \\ Estado de Mato Grosso (UNEMAT), Campus de Alta Floresta \\ ${ }^{2}$ Professora Doutora da Faculdade de Ciências Biológicas e Agrárias, UNEMAT, \\ Campus de Alta Floresta; Curadora do Herbário da Amazônia Meridional (HERBAM) \\ ${ }^{3}$ Professor Doutor da Faculdade de Ciências Biológicas e Agrárias, UNEMAT, \\ Campus de Alta Floresta; HERBAM; e-mail: ferbiobot@gmail.com
}

Recebido em: 03/10/2016 - Aprovado em: 21/11/2016 - Publicado em: 05/12/2016 DOI: 10.18677/EnciBio_2016B_027

\begin{abstract}
RESUMO
O gênero Bauhinia L. possui distribuição Pantropical e encontra-se representado por 160 espécies e destas, 61 ocorrem no Brasil e 21 para o Estado do Mato Grosso. O gênero é reconhecido principalmente pela presença de folhas bilobadas, palminérvias, flores pentâmeras, zigomorfas, hipanto tubuloso, pétalas livres e fruto do tipo legume. Este trabalho teve como objetivo apresentar uma contribuição taxonômica ao estudo do gênero Bauhinia para o Mato Grosso, com o levantamento das espécies que ocorrem no estado no site Flora do Brasil, juntamente com estudo taxonômico para as novas ocorrências depositadas no Herbário da Amazônia Meridional (HERBAM). Foi realizado entre março e maio de 2016 com as descrições morfológicas feitas no Laboratório de Morfologia Vegetal do HERBAM, localizado no Campus Universitário de Alta Floresta (UNEMAT). As ilustrações foram realizadas no Laboratório de Sistemática Vegetal da Universidade Federal de Viçosa. As espécies Bauhinia conwayi Rusby. e B. rufa (Bong.) Steud são novas ocorrências para o Estado, a primeira espécie possui folhas bilobadas com lâminas 7,9-18,4 $x$ 2,9-8,5 cm, parte unida menor que $1 / 3$ do comprimento total, cartáceas a coriáceas, base arredondada a subtruncada, 9-11 nervuras, ápice acuminado com lobos divaricados entre $0,5-6 \mathrm{~cm}$ compr., enquanto que a segunda espécie possui folhas bilobadas com lâmina 5,6-15,5 x 4,2-17,7 cm, parte unida em 2/2-3/1 do comprimento total, cartáceas a coriáceas, base de arredondada a subtruncada, 1115 nérvea, nervura marginal inconspícua, ápice agudo a obtuso, com lobos divaricados entre 2-9,5 cm compr. O presente trabalho reforça a ampliação de 21 para 23 espécies de Bauhinia para o Mato Grosso.
\end{abstract}

PALAVRAS-CHAVE: Fabaceae, flora Matogrossense, pata-de-vaca.

\section{TAXONOMIC CONTRIBUTION TO THE STUDY OF GENUS Bauhinia L. (LEGUMINOSAE) IN MATO GROSSO STATE, BRAZIL}

\author{
ABSTRACT \\ The genus Bauhinia L. has Pantropical distribution and is represented by 160 \\ species and from these, 61 are found in Brazil and 21 for Mato Grosso State. Genus
}


is mainly recognized by the presence of bilobed leaves, palmately netted, pentamerous flowers, zygomorphic, tubuloso hypanthium, free petals and fruits of the legume type. This study aimed to present a taxonomic contribution to the Bauhinia genus study to Mato Grosso, with the survey of the species that occur in the State on the site Flora of Brazil, along with taxonomic study for new records deposited in the Herbarium of the Southern Amazon (HERBAM). It was conducted between March and May, 2016 with the completion of morphological descriptions in Plant Morphology Laboratory HERBAM, located in the University Campus of Alta Floresta (UNEMAT). The illustrations were done in Systematics Laboratory vegetable Federal University of Viçosa. The species B. conwayi Rusby. and B. rufa (Bong.) Steud are new records for the State, the first species has bilobed leaves with blades from 7,9 to $18,4 \times 2,9$ to $8,5 \mathrm{~cm}$, less united party $1 / 3$ length total cartáceas the leathery, rounded base subtruncada, 9-11 ribs, apex acuminate with divaricados wolves between 0.5-6 cm long., while the second species has bilobed leaves with blade 5,6 to $15,5 \times 4,2$ to $17,7 \mathrm{~cm}$, united part $2 / 2-3 / 1$ full length, leathery to leathery, rounded base to subtruncada, 11-15 nérvea, inconspicuous marginal ridge, apex acute to obtuse, with divaricados between wolves 2 to $9,5 \mathrm{~cm}$ long. This study reinforces the expansion from 21 to 23 species of Bauhinia to Mato Grosso State.

KEYWORDS: Fabaceae, flora Matogrossense, pata-de-vaca.

\section{INTRODUÇÃO}

O Estado de Mato Grosso possui uma das floras mais ricas do Brasil com ocorrência de 181 famílias, 1.362 gêneros e 5.896 espécies de Angiospermas, destacando-se Leguminosae com 120 gêneros e 607 espécies (FLORA DO BRASIL 2020 EM CONSTRUÇÃO, 2016). Os maiores gêneros de Leguminosae no Brasil em espécies são Mimosa L. (323 spp), Chamaecrista Moench (253 spp), Inga Mill. (131 spp), Swartzia Schreb. (100 spp), Senna Mill. (80 spp), Calliandra Benth. (74 spp), Machaerium Pers. (73 spp) e Bauhinia L. (61 spp).

O Estado de Mato Grosso com 903.378,292 km² possui três dos mais importantes biomas - Amazônia, Cerrado e Pantanal; a Amazônia ocupa cerca de $50 \%$ do território, principalmente no norte do Estado, referência em biodiversidade mundial; o Cerrado cobre 38,29\%, localizado nas depressões de Alto Paraguai Guaporé, Sul e Sudeste do Planalto dos Parecis; e, o Pantanal, com 7,2\% da área do Estado, considerado a maior área alagável do planeta (MT, 2016). No Estado, destacam-se em número de espécies os gêneros: Mimosa L. com 51 espécies e Chamaecrista Moench com 48 espécies, Bauhinia L. está representado por 21 espécies (FLORA DO BRASIL 2020 EM CONSTRUÇÃO, 2016; VAZ, 2016a).

O gênero Bauhinia L. estabelecido em 1753 por Carl von Linnaeus possui distribuição pantropical, com cerca de 160 espécies, e pertence à tribo Cercideae Bronn, considerada a mais basal da família (LEWIS \& FOREST, 2005).

Nos últimos cinquenta anos, o gênero Bauhinia tem sido mais frequentemente reconhecido como um táxon amplamente circunscrito incluindo Phanera (Lour.) Wunderlin, K. Larsen \& S.S. Larsen como subgênero, no entanto, recentemente o subgênero foi reestabelecido com status genérico baseado em evidências moleculares e morfológicas como lianas com gavilhas, flores com uma pétala diferenciada e legumes samaróides (MACKINDER \& CLARK, 2014).

Os gêneros Bauhinia e Phanera Lour. assemelham-se pelas folhas bilobadas com nervação palminérvia (QUEIROZ, 2009). Bauhinia é caracterizado como: árvores, arbustos ou subarbustos; gavinhas ausenses; folhas alternas, espilaradas ENCICLOPÉDIA BIOSFERA, Centro Científico Conhecer - Goiânia, v.13 n.24; p.307 2016 
ou dísticas inteiras, bilobadas, bipartidas ou bifolioladas, estípulas caducas ou persistentes, estipelas ausentes, nectário intra-estipular às vezes presente, nervação palminérvia, 3-13 nervuras; inflorescências racemosas, paniculadas ou pseudoracemosas, terminais ou axilares, flores pentâmeras, zigomorfas ou actinomorfas, hipanto tubuloso ou campanulado, cálice dialissépalo ou gamossépalo, corola dialipétala, branca, rosada ou avermelhada, principalmente, androceu iso a heterodínamo, dialistêmone, 10 estames, ovário uniovulado a pluriovulado; fruto legume ou legume samaróide (VAZ \& TOZZI, 2003).

O gênero Phanera, com 24 espécies no Brasil distingue-se de Bauhinia pelo hábito lianescente, presença de gavinhas nas axilas, flores principalmente com hipanto obsoleto e cálice campanulado com lobos regulares (QUEIROZ, 2009; VAZ, 2016b) e legume típico. No Brasil ainda tem oito espécies de Schnella Raddi (VAZ, 2016c), desmembradas de Bauhinia L. e dentre as características distintivas estão os frutos legumes samaróides com uma ou duas sementes.

Várias espécies da tribo são conhecidas no Brasil como pata de vaca, pata de boi, unha de vaca, unha de boi, casco de vaca, mororó e árvore orquídea, usadas como ornamental, para arborização urbana, reflorestamento de áreas degradadas, medicinal, madeira para pequenas construções, ferramentas, estacas e moirões, combustível, além da casca usada para fazer cordas (LEWIS \& FOREST, 2005; LORENZI, 2009; FERNANDES et al., 2014).

Dentre as espécies de Cercideae no Brasil, apenas a espécie $B$. integerrima Mart. ex Benth. foi incluída no Livro Vermelho da Flora do Brasil (LIMA et al., 2013), na categoria Em Perigo (EN). Esta espécie é considerada endêmica do Estado da Bahia, sob domínio fitogeográfico da Floresta Atlântica, em uma área de $552 \mathrm{Km}^{2}$ e estima-se o declínio na qualidade de hábitat desta espécie devido à intensa atividade agrícola da região. Os mesmos autores mencionam as espécies $B$. marginata (Bong.) Steud., Phanera grazielae (Vaz) Vaz e P. smilacina (Schott) Vaz, dentre as espécies da flora brasileira não ameaçada, de interesse para pesquisa e conservação, sendo as duas primeiras devido apresentarem distribuição geográfica restrita e deficiência de dados e a terceira espécie por apresentar apenas deficiência de dados.

No entanto, mais espécies da tribo precisam ser avaliadas nas próximas edições do Livro Vermelho da Flora do Brasil embasada na riqueza, endemismo e importância econômica das espécies no país. Por outro lado, exige um esforço em conjunto entre diferentes profissionais na busca de maior detalhamento da distribuição geográfica das espécies, da qualidade dos hábitats, quantidade das populações e uso das espécies.

Este trabalho teve por objetivo realizar o estudo taxonômico das novas ocorrências de Bauhinia L. para o Estado de Mato Grosso, realizando-se descrições morfológicas e apresentando comentários taxonômicos e informações sobre a distribuição geográfica das espécies.

\section{MATERIAL E MÉTODOS}

O trabalho foi realizado entre março e junho de 2016, a partir da análise morfológica das exsicatas de Bauhinia depositadas no HERBAM. O sistema de classificação foi baseado em LEWIS et al. (2005) e as novas ocorrências no trabalho de FERNANDES et al. (2015). As diagnoses morfológicas foram realizadas no Laboratório de Morfologia Vegetal do Herbário da Amazônia Meridional (HERBAM).

O HERBAM está localizado na Universidade do Estado de Mato Grosso, ENCICLOPÉDIA BIOSFERA, Centro Científico Conhecer - Goiânia, v.13 n.24; p.308 2016 
Campus Universitário de Alta Floresta e foi registrado na Rede Brasileira de Herbários em 2007; possui atualmente cerca de $500 \mathrm{~m}^{2}$ e 14000 exsicatas depositadas, oriundas de diversas tipologias florestais do Estado (SOARES LOPES, 2015), estando entre as principais coleções botânicas de Mato Grosso (GASPER et al., 2016).

A terminologia empregada nas diagnoses está disponível em FERNANDES \& GARCIA (2014). As ilustrações foram realizadas no Herbário VIC, da Universidade Federal de Viçosa.

\section{RESULTADOS E DISCUSSÃO}

O gênero Bauhinia está representado por 21 espécies no Estado do Mato Grosso (Quadro 1), destas, oito possuem exsicatas depositadas no HERBAM. Segundo FERNANDES et al. (2015) as espécies de Bauhinia depositadas no HERBAM são: $B$. acreana Harms, B. aculeata L., B. conwayi Rusby, B. longicuspis Benth., B. longifolia (Bong.) Steud., B. pulchella Benth., B. rufa (Bong.) Steud. e B. ungulata $L$. Segundo os mesmos autores, as espécies $B$. conwayi Rusby e $B$. rufa (Bong.) Steud. não são citadas para o Mato Grosso no site Flora do Brasil, tornandose então novas ocorrências para o Estado. As duas espécies foram coletadas no Norte do Estado, a partir da intensificação de expedições científicas na região, advindos de trabalhos de conclusão de curso e nos programas de resgate de flora para coleção científica da Usina Hidrelétrica de Energia Teles Pires.

QUADRO 1. Espécies de Bauhinia (Leguminosae, Caesalpinioideae) com ocorrência no Estado de Mato Grosso, Brasil. Biomas em Mato Grosso: Am=Amazonas, Ce=Cerrado, Pa-Pantanal. ${ }^{1}$ Novas ocorrências para Mato Grosso (FERNANDES et al., 2015).

\begin{tabular}{|l|c|c|}
\hline Espécies & Distribuição no Brasil & $\begin{array}{c}\text { Biomas } \\
\text { em MT }\end{array}$ \\
\hline B. acreana Harms & AC, AM, PA, RO, CE, \\
MA, MT & Am \\
\hline B. bauhinioides (Mart.) J.F.Macbr. & BA, MA, PI, MS, MT, \\
MG, SP & Pa \\
\hline B. caloneura Malme & RO, TO, BA, PI, GO, MS, MT, MG, & Am, Ce \\
\hline B. campestris Malme & MT & Ce \\
\hline B. cheilantha (Bong.) Steud. & MS, MT & Ce \\
\hline B. conwayi Rusby & SE, MS, & Ce \\
\hline B. corniculata Benth. & MT, MG, SP & \\
\hline B. cupulata Benth. & AC, AM, PA, TO, MS, & Am, Pa \\
\hline B. curvula Benth. & MT & Am, \\
\hline B. goyazensis Harms & PA, TO, BA, MA, PI, GO, MT & Am, Ce \\
\hline B. holophylla (Bong.) Steud. & TO, DF, GO, MS, MT, MG & Am, Ce \\
\hline B. longicuspis Benth. & TO, DF, GO, MT & Ce \\
\hline
\end{tabular}

ENCICLOPÉDIA BIOSFERA, Centro Científico Conhecer - Goiânia, v.13 n.24; p.309 2016 


\begin{tabular}{|c|c|c|}
\hline B. longifolia (Bong.) Steud. & $\begin{array}{c}\text { PA, RO, BA, DF, GO, MS, MT, ES, } \\
\text { MG, RJ, } \\
\text { SP, PR }\end{array}$ & $\mathrm{Ce}$ \\
\hline B. longipedicellata Ducke & $\mathrm{PA}, \mathrm{RO}, \mathrm{MT}$ & $\mathrm{Am}$ \\
\hline B. marginata (Bong.) Steud. & MS, MT, SP & $\mathrm{Ce}$ \\
\hline B. mollis (Bong.) D.Dietr. & TO, GO, MS, MT, MG & $\mathrm{Ce}, \mathrm{Pa}$ \\
\hline B. pentandra (Bong.) D.Dietr. & $\begin{array}{c}\text { TO, AL, BA, CE, MA, PB, PE, PI, } \\
\text { RN, SE, } \\
\text { GO, MS, MT, MG, SP }\end{array}$ & $\mathrm{Ce}$ \\
\hline B. platypetala Burch. ex Benth. & PA, TO, MA, PI, GO, MT & $\mathrm{Am}, \mathrm{Ce}$ \\
\hline B. pulchella Benth. & $\begin{array}{c}\mathrm{PA}, \mathrm{RO}, \mathrm{TO}, \mathrm{BA}, \mathrm{CE}, \mathrm{MA}, \mathrm{PE}, \mathrm{PI}, \\
\mathrm{RN}, \mathrm{GO}, \\
\mathrm{MS}, \mathrm{MT}, \mathrm{MG}\end{array}$ & $\mathrm{Am}, \mathrm{Ce}$ \\
\hline B. rufa (Bong.) Steud. & $\mathrm{DF}, \mathrm{GO}, \mathrm{MT}^{1}$ & $\mathrm{Ce}$ \\
\hline B. ungulata $\mathrm{L}$. & $\begin{array}{c}\text { AC, AM, AP, PA, RO, RR, TO, BA, } \\
\text { CE, MA, } \\
\text { PI, DF, GO, MS, MT, } \\
\text { MG, RJ, SP }\end{array}$ & $\mathrm{Am}, \mathrm{Ce}$ \\
\hline B. vespertilio S.Moore & MA, GO, MT & $\mathrm{Ce}$ \\
\hline
\end{tabular}

As 21 espécies que ocorrem no Mato Grosso, ocorrem predominantemente no Cerrado e Amazônia, poucas no Pantanal (Quadro 1). Apenas B. caloneura Malme é endêmica do Estado, com ocorrência no Cerrado. Segundo VAZ \& TOZZI (2003), trata-se de uma espécie restrita a áreas de Cuiabá e Nova Xavantina, mas a espécie é pouco conhecida bem como as suas afinidades.

Bauhinia conwayi Rusby, Bull. New York Bot. Gard. 8(28): 92-93. 1912. (Figura 1).

Arbustos. Folhas bilobadas, lâmina 7,9-18,4 x 2,9-8,5 cm, lobos concrescidos menores que $1 / 3$ do comprimento total, cartáceas a coriáceas, base arredondada a subtruncada, 9-11 nervuras, ápice acuminado com lobos divaricados entre 0,5-6 cm compr.; face adaxial glabra; face abaxial esparsamente pubescente, tricomas glandulares esparsamente presentes, nervuras primárias proeminentes, secundárias mais ou menos proeminentes, terciárias pouco proeminentes; pecíolo 0,7-1,7 cm compr., glabescente; estípulas caducas; nectários extraflorais estreitamento piriformes, 1,2 mm compr., excertos. Inflorescência 9-25 cm compr., tomentosa. Botão floral 3,5-8 cm compr., reto a levemente curvo, ápice agudo, segmentos irregulares na pré-antese. Flores com pedicelo 0,5-1,7 cm compr., brácteas 0,15-0,1 cm compr., hipanto cilíndrico, 0,9-1,6 cm compr., internamente glabro; cálice com 5 lobos, ondulados, $5-7,5 \mathrm{~cm}$ compr., corola 5 pétalas, lineares, longamente acuminadas, 1,8-2 cm compr., externamente glabras; estames 10 , filetes $3,2-8,6 \mathrm{~cm}$ compr., glabros, anteras $0,7-1,3 \mathrm{~cm}$ compr., iguais, lineares; gineceu $3,8-11,5 \mathrm{~cm}$ compr., estipe 1,6-4,7 cm compr., tomentoso a esparso tomentoso, sem tricomas glandulares, ovário tomentoso, estilete glabro, estigma claviforme. Fruto legume, estipe 3,5-4,6 cm compr., valvas 7,4-13,8 x 0,8-1,3 cm., base aguda, ápice cuspidado, esparsamente puberulento externamente; 9-17 sementes, 0,5-0,7 x 0,4 $\mathrm{cm}$, enegrecidas, elípticas, raramente circulares. 
Comentários: B. conwayi é caracterizada por apresentar lâmina foliar grande, entre $9-26 \mathrm{~cm}$ de comprimento, bilobadas, lobos concrescidos em até $1 / 3$ do comprimento total, divaricados nas folhas maiores entre 6 e $12 \mathrm{~cm}$, eixo da inflorescência tomentoso a velutino, botão floral com ápice nunca acuminado ou caudado, pétalas lineares e valvas do fruto pubérulos e ovário sem tricomas glandulares (VAZ \& TOZZI, 2003). Segundo as mesmas autoras, a espécie mais relacionada é $B$. longicuspis Benth., mais se diferencia por apresentar folhas inteiras, se bilobadas, os lobos concrescidos em 2/3 do comprimento ou mais e eixo da inflorescência pubérulo. Ocorre na Bolívia e Brasil (VAZ \& TOZZI, 2003). No Brasil, existe registro de ocorrência para os Estados do Acre, Pará e Rondônia, sob domínio fitogeográfico da Amazônia em floresta de terra firme e floresta de várzea (VAZ, 2016a). No Mato Grosso foi coletada no município de Paranaíta.

Material selecionado: BRASIL. MATO GROSSO: Paranaíta, UHE Teles Pires (918'33"S, 56ㄴㄱ'40"W) 28/XII/2011), fl., C.R.A. Soares, L. Silvino, F.C. Benetti, J.R.R. Silva, N.F. Moura 4936 (HERBAM).

Bauhinia rufa (Bong.) Steud., Nomencl. Bot. (ed. 2) 1(1-2): 192. 1840. (Figura 1).

Arbustos. Folhas bilobadas, lâmina 5,6-15,5 x 4,2-17,7 cm, lobos concrescidos em 2/2-3/1 do comprimento total, cartáceas a coriáceas, base de arredondada a subtruncada, 11-15 nérvea, nervura marginal inconspícua, ápice agudo a obtuso, com lobos divaricados entre 2-9,5 cm compr.; face superior glabra; face inferior opaca, tricomas glandulares, nervuras primárias e secundárias bem proeminentes, terciárias proeminentes; pecíolo robusto $1,3 \mathrm{~cm}$ compr., viloso-tomentoso; estípulas 1-3 x 1,5-2 mm, ovada a amplamente ovada, tomentosa externamente; nectários extraflorais, 2-3 mm compr., estreitamento piriforme, excertos. Inflorescência 7-16 $\mathrm{cm}$ compr., tomentosa. Botão floral 4-9 cm compr., ápice agudo a arredondado. Flores com pedicelo 0,4-2 cm compr., brácteas 0,2-0,4 cm compr., hipanto subcilíndrico, 0,9-2,8 cm compr., internamente glabro a puberulento; cálice com 5 lobos, ondulados a retorcidos e espiralados $3,2-16,5 \mathrm{~cm}$ compr.; corola 5 pétalas lineares, longamente acuminadas, 2,3-13,5 cm compr., externamente glabras; estames 10, anteras iguais, lineares, filetes 2,4-14,2 cm compr., glabros; anteras 0,9-2,7 cm compr.; gineceu 5-17 cm compr., região terminal tomentosa, sem tricomas glandulares, estigma claviforme, ovário tomentoso, estipe 1-3,1 cm compr., tomentoso. Frutos não observados.

Comentários: $B$. rufa distingue-se das espécies do complexo B. holophylla (Bong.) Steud. e B. longifolia (Bong.) Steud. por apresentar folhas coriáceas, curtamente bilobadas, obtusas a arredondadas, parte superior levemente bulada, ramos, pecíolos, inflorescência e botão floral com indumento ferrugíneo, velutino a hirsuto e estípulas lineares a oblongo agudas (VAZ \& TOZZI, 2003). Segundo as mesmas autoras, $B$. longifolia apresenta folhas com lobos agudos a obtusos, tênuecartáceos a sub-coriáceas e estípulas ovadas a ovado-lanceoladas. Segundo VAZ (2016a) ocorre apenas nos Estados de Minas Gerais, Goiás e Distrito Federal, nas fitofisionomias campo rupestre e cerrado lato sensu, no domínio do Cerrado. 


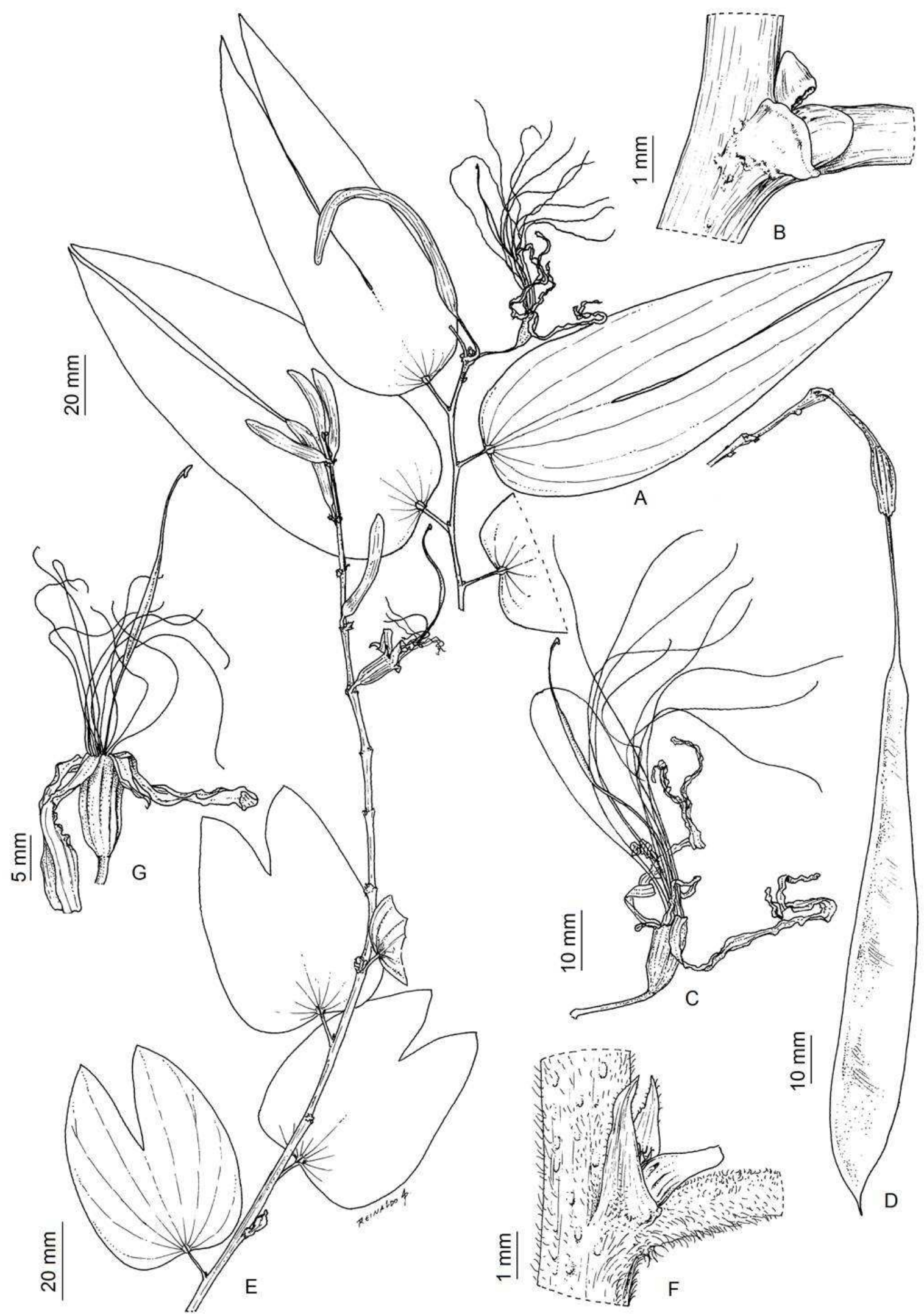

FIGURA 1. Bauhinia conwayi: A, parte do ramo com folha; $B$, estípulas e nectário extraflorais internamente; C, flor; D, fruto. Bauhinia rufa: $\mathrm{E}$, parte do ramo com folha; F, estípulas e nectário extrafloral internamente; G, flor. Ilustração: Reinaldo Pinto. 
Material examinado: BRASIL. MATO GROSSO: Alta Floresta, Fazenda Modelo, 19/V/2007, fl. J.A.M. Melo, L. Rodrigues, J.A. Pedroga 2 (HERBAM). Paranaíta, UHE Teles Pires (918'57"S, 5647'51'W), 12/IV/2012, fl. C.R.A. Soares, M. Barros, F.F. Cazine, E. Benetti, V.A. Moura 5983 (HERBAM).

\section{CONCLUSÃO}

Os caracteres de $B$. conwayi e $B$. rufa estão de acordo com a última revisão taxonômica realizada para o gênero. Este trabalho reforça a ampliação de 21 para 23 espécies de Bauhinia para a flora de Mato Grosso. O trabalho demostra a importância das coleções botânicas para o melhor conhecimento da flora, bem como a intensificação de coletas em locais do Estado mato-grossense com poucas coletas realizadas, como a região norte.

\section{REFERÊNCIAS}

FERNANDES, J. M.; GARCIA, F. C. P.; AMOROZO, M. C. M.; SIQUEIRA, L. C.; MAROTTA, C. P. B.; CARDOSO, I. M. Etnobotânica de Leguminosae entre agricultores agroecológicos na Floresta Atlântica, Araponga, Minas Gerais, Brasil. Rodriguésia, v. 65, n. 2, p.539-554, 2014.

FERNANDES, J. M.; SOARES-LOPES, C. R. A.; RIBEIRO, R. S.; SILVA, D. R. Leguminosae no acervo do Herbário da Amazônia Meridional, Alta Floresta, Mato Grosso. Enciclopédia Biosfera, v. 11, n. 21, p. 2272-2293, 2015.

FLORA do Brasil 2020 em construção. Jardim Botânico do Rio de Janeiro. Disponível em: < http://floradobrasil.jbrj.gov.br/ >. Acesso em: 29 Set. 2016.

FERNANDES, J. M; GARCIA, F. C. P. Expanding the description of Bionia bella Mart. ex Benth. (Leguminosae, Papilionoideae). Acta Botanica Brasilica, v. 28, n. 2, p. 141-146, 2014.

GASPER, A. L.; VIEIRA, A. O. S.; SOARES LOPES, C. R. A.; BIGIO, N.; MACEDO, G. E. L. Rede Brasileira de Herbários. Disponível em: http://www.botanica.org.br/rbh. Acessado em: 29 de setembro de 2016.

LEWIS, G. P.; FOREST, F. Cercideae Bronn. In: LEWIS, G. P.; SCHRIRE, B.; MACKINDER, B.; LOCK, M. (eds.). Legumes of the world. Kew: Royal Botanic Gardens, pp.57-67, 2005.

LEWIS, G. P.; SCHRIRE, B.; MACKINDER, B.; LOCK, M. Legumes of the world. Kew: Royal Botanic Gardens, 577p., 2005.

LIMA, H. C.; SOUZA, E. R.; TOZZI, A. M. G. A.; PEREZ, A. P. F.; FlORES, A. S.; SARTORI, A. L. B.; VAZ, A. M. S. F.; FILARDI, F.; FERNANDES, F. M.; GARCIA, F. C. P.; IGANCI, J. R. V.; FERNANDES, J. M.; VALLS, J. F. M.; LIMA, L. C. P.; COSTA, L. C.; QUEIROZ, L. P.; MORIM, M. P.; NUNES, N. L. A.; QUEIROZ, R. T.; MIOTTO, S. T. S.; DUTRA, V. F.; MANSANO, V. F.; SOUZA, V. C.; MESSINA, T.; KUTSCHENKO, D. C.; PENEDO, T. S. A.; VALENTE, A. M. Fabaceae/ Leguminosae. In: Martinelli, G.; Moraes, M. A. (orgs.). Livro vermelho da flora do Brasil. Instituto de Pesquisas Jardim Botânico do Rio de Janeiro, Rio de Janeiro. 1 ENCICLOPÉDIA BIOSFERA, Centro Científico Conhecer - Goiânia, v.13 n.24; p.313 2016 
ed., pp. 516-548, 2013.

LORENZI, H. Árvores brasileiras: manual de identificação e cultivo de plantas do Brasil. Nova Odessa, São Paulo: Instituto Plantarum, 384p., 2009.

MACKINDER, B. A.; CLARK, R. 2014. A synopsis of the Asian and Australasian genus Phanera Lour. (Cercideae: Caesalpinioideae: Leguminosae) including 19 new combinations. Phytotaxa, v. 166, n. 1, p. 049-068, 2014.

MT. Geografia. Disponível em: http://www.mt.gov.br/geografia. Acessado em 15 de janeiro de 2016.

QUEIROZ, L. P. Leguminosas da Caatinga. Feira de Santana: Universidade Estadual de Feira de Santana, 443p., 2009.

SOARES LOPES, C. R. A. Herbário da Amazônia Meridional. Unisanta Bioscience, v. 4 , n. 6 , p.36-39, 2015.

VAZ, A. M. S. F. Bauhinia in Lista de Espécies da Flora do Brasil. Jardim Botânico do Rio de Janeiro. Disponível em: <http://floradobrasil.jbrj.gov.br/jabot/floradobrasil/FB82684>. Acesso em: 22 Jan. 2016a.

VAZ, A. M. S. F. Phanera in Lista de Espécies da Flora do Brasil. Jardim Botânico do Rio de Janeiro. Disponível em: <http://floradobrasil.jbrj.gov.br/jabot/floradobrasil/FB25673>. Acesso em: 22 Jan. 2016b.

VAZ, A. M. S. F. Schnella in Lista de Espécies da Flora do Brasil. Jardim Botânico do Rio de Janeiro. Disponível em: <http://floradobrasil.jbrj.gov.br/jabot/floradobrasil/FB129669>. Acesso em: 22 Jan. 2016c.

VAZ, A. M. S. F.; TOZZI, A. M. G. A. Bauhinia ser. Cansenia (Leguminosae: Caesalpinioideae) no Brasil. Rodriguésia, v. 54, n. 83, p. 55-143, 2003. 